\title{
Ausbau von Wärmenetzen vs. energetische Sanierung? - Umgang mit konkurrierenden Strategien zur Umsetzung der ,Wärmewende“ auf kommunaler Ebene
}

\author{
Susanne Schubert ${ }^{1}$
}

Eingegangen: 29. März 2015 / Angenommen: 12. April 2016 / Online publiziert: 25. Mai 2016

(c) Springer-Verlag Berlin Heidelberg 2016

Zusammenfassung Strategien zur Integration erneuerbarer Energien in die Wärmeversorgung von Städten umfassen oftmals den Auf- oder Ausbau von Wärmenetzen. Die gleichzeitige energetische Sanierung des Gebäudebestands zur Reduktion der Nachfrage wurde verschiedentlich in der Literatur als eine mit Wärmenetzen konkurrierende Strategie beschrieben, da netzgebundene Systeme in der Regel auf einen vergleichsweise hohen Verbrauch angewiesen sind. Im Fokus dieses Beitrags steht die Frage, inwieweit es sich vor dem Hintergrund der klimapolitischen Zielsetzungen um zwei inkompatible und damit konkurrierende Strategien zur Umsetzung der ,Wärmewende“ handelt. Aus den hier betrachteten Fallstudien wird deutlich, wie auf kommunaler Ebene mit diesen Strategien umgegangen wird. Es zeigen sich sowohl Konkurrenzen wie auch Anpassungsmöglichkeiten und Synergien zwischen beiden Strategien.

Schlüsselwörter Wärmeversorgung · Energetische Sanierung $\cdot$ Energiewende $\cdot$ Schweiz

\section{Strengthening heating grids vs. energetic renovation? - Handling of competing strategies for implementing the thermal energy transformation on the municipal level}

Abstract Strategies to integrate renewable energies into urban heat supply are often based on the installation and development of district heating grids. At the same time

Dr.-Ing. Susanne Schubert

s.schubert@iwar.tu-darmstadt.de

1 Fachgebiet Raum- und Infrastrukturplanung, TU Darmstadt, Franziska-Braun-Straße 7, 64287 Darmstadt, Deutschland the energetic transformation of the building stock to reduce energy demand has been described as a competing strategy to grid-based systems, due to the characteristics of heating grids which generally are dependent on a comparatively high energy demand. Against this background the article asks, how far heating grids and energetic renovation are incompatible and therefore competing strategies to reach the thermal energy transformation. Based on results from case studies the article lines out, how these strategies are implemented at the municipal level. It is shown that competition as well as adaption and synergies are ways to cope with the existence of the two strategies.

Keywords Heat supply · Energetic renovation · Energy transformation $\cdot$ Switzerland

\section{Einleitung}

Die Steigerung der Energieeffizienz und die Erhöhung des Anteils erneuerbarer Energien sind Eckpunkte der gegenwärtigen Energie- und Klimaschutzpolitik. Die energiepolitischen Ziele Deutschlands erfordern nicht nur in der Stromversorgung eine tief greifende Transformation, sondern auch einen radikalen Umbau der Wärmeversorgung. Während bei der Integration erneuerbarer Energien in die Stromversorgung bereits deutliche Erfolge erzielt werden konnten, hinkt die Energiewende im Bereich der Wärmeversorgung hinterher, sowohl hinsichtlich des Ziels einer Reduktion der Energienachfrage als auch beim Ziel einer Erhöhung des Anteils erneuerbarer Energien an der Wärmeerzeugung. Die Herausforderungen angesichts der Wechselwirkungen und Abhängigkeiten zwischen der Wärmeversorgung und der Raumstruktur zeigen sich vor allem in den Städten, wo hinsichtlich der spezifischen Gebäude- 
Tab. 1 Übersicht über die Fallstudien

\begin{tabular}{ll}
\hline Frankfurt am Main & - Verdichtung im Fernwärmenetz, Temperaturabsenkung \\
& - Dezentrale Nahwärme mit Blockheizkraftwerken \\
& - „Passivhaushauptstadt“ \\
Basel & - Verdichtung im Fernwärmenetz, Neuanschluss an den Rändern, Temperaturabsenkung \\
& - Intensive Förderung energetischer Sanierung \\
Bern & - Umfangreicher Ausbau der Fernwärme, v. a. in UNESCO-Welterbe Altstadt \\
& - Dezentrale Nahwärme zur Nutzung erneuerbarer Energien, v. a. Erdwärme \\
Winterthur & - Verdichtung im Fernwärmenetz, Neuanschluss an den Rändern \\
& - Dezentrale Nahwärme zur Nutzung erneuerbarer Energien, v. a. Energieholz \\
\hline
\end{tabular}

struktur und der Verfügbarkeit teilweise örtlich gebundener, erneuerbarer Energieträger spezifische Lösungen gefunden werden müssen.

Gerade im städtischen Raum und insbesondere im Bestand stellt sich die Integration erneuerbarer Energien in die Wärmeversorgung oftmals schwierig dar, lässt sich nur schwer in Einzelanlagen in jedem Gebäude realisieren und ist in vielen Fällen auf Wärmenetze angewiesen. Wärmenetze wiederum sind als netzgebundene Systeme mit hohen Fixkosten in der Regel von einer vergleichsweise hohen Nachfragedichte abhängig, um ökonomisch betrieben werden zu können (Paar/Herbert/Pehnt et al. 2013: 18). Damit scheint hier ein Zielkonflikt zwischen der Energieversorgung über Wärmenetze und dem gleichzeitigen Vorhaben einer energetischen Sanierung zu bestehen; daraus würde eine Konkurrenz zwischen den Strategien der Effizienzsteigerung auf der Nachfrageseite einerseits und der Versorgung über Wärmenetze andererseits folgen. Dieser Zielkonflikt ist in der einschlägigen Literatur bereits aufgegriffen worden (Späth 2005; Wolff/Jagnow 2011; Magnusson 2012).

Jedoch muss der beschriebene Zielkonflikt nicht in jedem Fall in eine Konstellation konkurrierender Strategien führen. Stattdessen bestehen auch Möglichkeiten einer Koexistenz beider Strategien, die sich in verschiedener Weise zeigen. In diesem Beitrag soll anhand kommunaler Fallstudienergebnisse gezeigt werden, wie auf der Umsetzungsebene mit den Verbindungen und möglichen Widersprüchen zwischen den Zielen und Strategien des klimafreundlichen Umbaus der Wärmeversorgung konkret umgegangen wird. Dabei steht die Frage im Mittelpunkt, ob und wie sich eine Konkurrenz zwischen netzgebundenen Wärmeversorgungsstrategien und der Reduktion der Energienachfrage durch energetische Sanierung zeigt bzw. inwieweit eine Koexistenz beider Strategien möglich ist.

Methodisch basiert dieser Artikel auf der Auswertung wissenschaftlicher Literatur sowie hinsichtlich der Ergebnisse der kommunalen Fallstudien auf der Auswertung von Planungsdokumenten, vor allem Energiekonzepten und Energiefachplänen, sowie insgesamt neun leitfadengestützten Experteninterviews, davon drei mit Vertretern kommunaler Energiefachstellen und sechs mit Vertretern der kommunalen Energieversorgung.

Die vier hier betrachteten Fallbeispiele beinhalten eine deutsche Stadt und drei Schweizer Städte:

- Frankfurt am Main,

- Winterthur,

- Bern,

- Basel.

Alle vier Städte sind Großstädte (mit mehr als 100.000 Einwohnern), deren Ziele, Planungen und Strategien für den Umbau der Wärmeversorgung im Zuge der Energiewende als ambitioniert gelten. Alle Städte setzen einerseits deutlich auf netzgebundene Versorgungsstrategien und zugleich auch auf eine deutliche Reduktion der Energienachfrage. Alle diese Städte haben zudem Energiekonzepte oder Energiefachpläne für die gesamtstädtische Ebene (teilweise mit Konkretisierungen für die Quartiersebene), die sowohl den Ausbau und die Transformation der Wärmenetze adressieren als auch Vorhaben der energetischen Sanierung ausweisen. Frankfurt am Main ist seit vielen Jahren sowohl organisatorisch als auch mit seinen Erfolgen und ambitionierten Plänen Vorreiter beim klimafreundlichen Umbau der Wärmeversorgung (Stadt Frankfurt am Main, Dezernat Umwelt und Gesundheit und Dezernat Planen, Bauen, Wohnen und Grundbesitz 2007: 3). Daneben bieten die Schweizer Städte durch die hier verankerte Energiefachplanung auf kommunaler Ebene ebenfalls interessante und gut dokumentierte Beispiele für das Aufeinandertreffen des Aus- und Umbaus von Wärmenetzen bei gleichzeitiger energetischer Sanierung (Berger/Genske/Hüsler et al. 2011; Stadt Winterthur, Umwelt- und Gesundheitsschutz 2011; Stadt Bern, Direktion für Sicherheit, Umwelt und Energie 2012) (vgl. auch Tab. 1).

Die konkreten Planungen und Projekte für den Ausbau und die Transformation der Wärmenetze und zugleich die energetische Sanierung in den Städten beziehen sich auf unterschiedliche Raum- und Siedlungsstrukturen. Dazu gehören die von zentralen Fernwärmenetzen versorgten innerstädtischen Gebiete (teilweise mit gemischter Nutzung, teilweise mit überwiegender Wohnnutzung) in verdichteter Bebauung, darunter Gründerzeitstrukturen und Quartiere aus 
den 1950er- bis 1970er-Jahren. Andererseits stehen gerade in Projekten für den Ausbau dezentraler Nahwärmenetze mit zugleich hohem Energieeffizienzstandard der Gebäude auch randstädtische Wohnquartiere aus den 2000er-Jahren und später errichtete Neubauquartiere im Fokus.

Die ausgewählten Städte und die betrachteten Planungen und Projekte innerhalb dieser Städte reflektieren nicht die Bandbreite aller denkbaren Raum- und Siedlungsstrukturen. In ihren Zielsetzungen und der Vielfalt ihrer Maßnahmen in der Wärmeversorgung geben sie aber einen guten Einblick, wie mit den Möglichkeiten konkurrierender oder koexistierender Strategien umgegangen wird.

Zunächst werden in Abschn. 2 die Zielsetzungen der Energiewende für die Wärmeversorgung und den Gebäudebestand erläutert und die Rahmenbedingungen für ihre Realisierung umrissen. Anschließend wird in Abschn. 3 mit Bezug auf die sozialwissenschaftliche Technikforschung sowie auf aktuelle Literatur zur Struktur und Veränderung der Wärmeversorgung und -nutzung im Kontext der politischen Zielsetzungen der konzeptionelle Rahmen für eine Konkurrenz oder Koexistenz zwischen dem Ausbau der Wärmenetze und energetischer Sanierung abgesteckt und drei Formen des Aufeinandertreffens dieser Strategien abgeleitet. Auf der Grundlage dieser Struktur werden in Abschn. 4 verschiedene Beispiele aus kommunalen Fallstudien diskutiert, die verdeutlichen, wie sich das beschriebene Phänomen auf der Umsetzungsebene in den Städten zeigt und wie damit umgegangen wird. Abschn. 5 fasst abschließend die Beobachtungen zusammen und verweist auf offene Fragen.

\section{Zielsetzungen und Rahmenbedingungen der Wärmewende}

Für die Energiewende in der Wärmeversorgung sind die energetische Sanierung des Gebäudebestands und die Steigerung des Anteils erneuerbarer Energien an der Wärmeerzeugung die entscheidenden Parameter, um die politisch definierten Ziele zu erreichen. Das Energiekonzept der Bundesregierung sieht vor, die Sanierungsrate von 1 Prozent auf 2 Prozent pro Jahr zu steigern und somit den Energiebedarf für die Wärmeversorgung um 20 Prozent bis 2020 und 50 Prozent bis 2050 gegenüber 2008 zu senken (Bundesministerium für Wirtschaft und Technologie 2010: 22). Das Erneuerbare-Energien-Wärme-Gesetz (EEWärmeG) formuliert als Zielsetzung einen Anteil von 14 Prozent erneuerbarer Energien an der Wärmeversorgung bis 2020 ( $\$ 1$ EEWärmeG). Das zuletzt 2012 aktualisierte Leitszenario für die Energiewende im Auftrag des Bundesministeriums für Umwelt, Naturschutz, Bau und Reaktorsicherheit zeigt langfristige Entwicklungspfade für die Energiewende auf und hat im Rahmen von vier Szenarien unterschiedliche Wege für das Ziel einer Treibhausgasreduktion um 80 Pro- zent bis 2050 definiert. In diesen Szenarien werden noch deutlich weitreichendere Ziele zugrunde gelegt. So könne bei entsprechenden Anstrengungen der Anteil erneuerbarer Energien an der Wärmeversorgung bis 205053 Prozent betragen, ausgehend von einer Reduktion der Energienachfrage um 57 Prozent bis 2050 (Nitsch/Pregger/Naegler et al. 2012: 63, 128). Wachsende Anteile regenerativer Wärmegewinnung und die Reduktion des Wärmebedarfs hängen somit zusammen, da eine Erhöhung des Anteils erneuerbarer Energien leichter $\mathrm{zu}$ erreichen ist, wenn der Energiebedarf zurückgeht. Beides sind Herausforderungen auf kommunaler Ebene und für die Stadtplanung, die beiden Themenfeldern und ihren Schnittstellen Rechnung tragen muss. Aber auch diese ehrgeizigeren Ziele liegen noch deutlich unter denen für die Stromversorgung und weisen damit auf die nur langsamen Fortschritte bei der Integration erneuerbarer Energien in die Wärmeversorgung und bei der energetischen Sanierung des Bestands hin (Löschel/Erdmann/Staiß et al. 2014: 13).

Ein Grund für die sich nur zögerlich einstellenden Erfolge ist, dass gerade im städtischen Raum die Transformation der Wärmeversorgung hin zu erneuerbaren Energien oftmals schwer zu realisieren ist. Anders als bei der Stromversorgung muss die Wärme nah am Ort des Verbrauchs erzeugt werden, da sie sich nicht ohne größere Verluste und nur unter hohem ökonomischem Aufwand über weite Strecken transportieren lässt. In deutschen Städten basiert die Wärmeversorgung überwiegend auf Erdgas, das in Einzelanlagen direkt im Gebäude der Nutzer zum Einsatz kommt (AG Energiebilanzen 2013: 5). Nimmt man die Ziele der Energiewende für die Wärmeversorgung ernst, so muss langfristig die Dominanz dieses Systems verringert und eine Alternative für den städtischen Raum gefunden werden. Zugleich lässt sich im dicht besiedelten, urbanen Raum eine Versorgung nicht allein über Einzelanlagen auf der Basis erneuerbarer Energien realisieren. Die Gründe reichen von Argumenten zum effizienten Einsatz der Technik über die Frage der Verfügbarkeit örtlich gebundener Energieträger und ausreichender Raumverfügbarkeit vor Ort bis hin zu Emissionsschutzbedenken beispielsweise hinsichtlich der dezentralen Verbrennung von Holz. Um einen relevanten Anteil erneuerbarer Energien an der Wärmeversorgung zu erreichen, sehen Nitsch/Pregger/Naegler et al. daher gerade im städtischen Raum einen großen Bedarf für den Ausbau netzgebundener Wärmeversorgung, vor allem über Nahwärmenetze. Nach ihren Abschätzungen soll die netzgebundene Wärmeversorgung deutlich zunehmen, während der Einsatz fossiler Brennstoffe, insbesondere in Einzelfeuerungen, sinken muss (Nitsch/Pregger/Naegler et al. 2012: 124 f., vgl. Abb. 1). Als Energieträger sollen dabei unterschiedliche erneuerbare Energien zum Einsatz kommen, darunter als wesentliche Stütze weiterhin Biomasse (die heute unter den erneuerbaren Energien an der Wärme- 
Abb. 1 Szenario Endenergieeinsatz für Wärme (Nitsch/ Pregger/Naegler et al. 2012: 13)

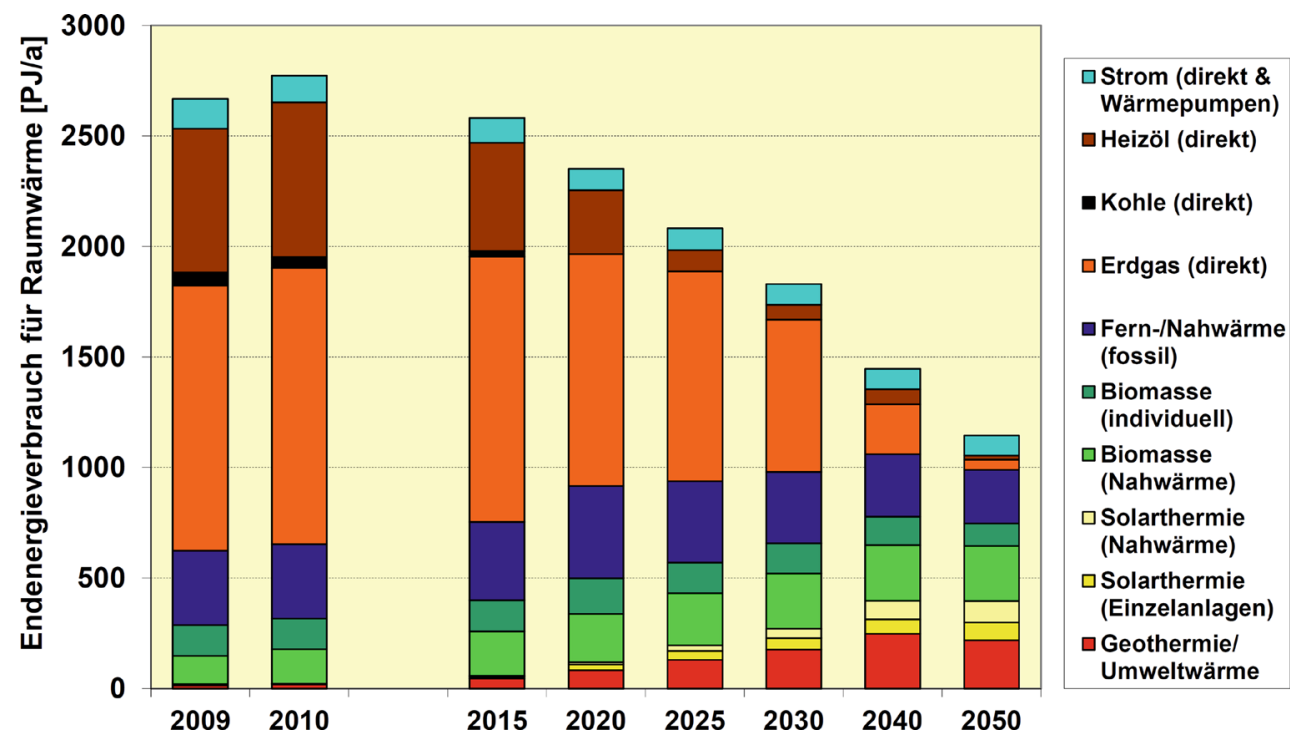

versorgung den mit Abstand größten Anteil hat), aber auch zunehmend Solarthermie, Geothermie und Umweltwärme, die ab ca. 2030 ähnlich hohe Anteile erreichen sollen wie die Biomasse (Nitsch/Pregger/Naegler et al. 2012: 125 f.; Löschel/Erdmann/Staiß et al. 2014: 16 f.).

Die Reduktion der Energienachfrage einerseits und die Steigerung des Anteils netzgebundener Wärmeversorgung andererseits scheinen im Widerspruch zu stehen, denn netzgebundene Systeme sind in der Regel auf gleichbleibende Verbrauchshöhen angewiesen. Sinkt die Wärmenachfrage, auch aufgrund verbesserter Gebäudeenergieeffizienz, und wird daher weniger Wärme verkauft, so geraten die Fernwärmeversorger ökonomisch unter Druck, da die hohen Kosten des Netzbetriebs auch bei geringerer Nachfrage bleiben (Späth 2005; Fischedick/Schüwer/Venjakob et al. 2006; Paar/Herbert/Pehnt et al. 2013: 19). Das wird bereits heute in zahlreichen Fernwärmenetzen sichtbar, denn der deutsche Wärmemarkt schrumpft aus vielfältigen Gründen (Paar/Herbert/Pehnt et al. 2013: 31 f.). Zum einen wirkt sich der Bevölkerungsrückgang in einigen Regionen auch auf die Energienachfrage aus, wobei diese Rückgänge teilweise durch die wachsende Wohnfläche pro Kopf sowie durch höhere Verbräuche der alternden Bevölkerung kompensiert werden (York 2007: 872). Auch die Häufung wärmerer Winter hat Auswirkungen auf die Energieverbräuche für Raumwärme; hinzu kommt die verbesserte Gebäudeenergieeffizienz (Graichen/Bürger/Gores et al. 2012: 6; Michelsen 2015: 922). Dies spiegelt sich auch im oben genannten Leitszenario wider, das von einem insgesamt deutlich sinkenden Wärmeverbrauch für Raumwärme (RW) und Warmwasser (WW) ausgeht, sodass der Haushaltssektor, der heute der größte Wärmenachfrager ist, bis 2050 weniger Energie für Wärme verbraucht als der Industriesektor für Prozesswärme (PW), während die Wärmenachfrage im Bereich Gewerbe, Handel, Dienstleistungen (GHD) bis dahin am deutlichsten sinkt (um $67 \%$ ) (Nitsch/Pregger/Naegler et al. 2012: 65, vgl. Abb. 2).

Inwieweit diese Erfolge in der energetischen Sanierung des Gebäudebestands und damit ein entsprechend deutlicher Rückgang der Wärmenachfrage aber tatsächlich realisiert werden können, bleibt abzuwarten. Bisher liegen die Ergebnisse hier deutlich hinter den Erwartungen und Zielen. Die Gründe sind vielfältig und liegen neben fehlendem Kapital und Know-how auch in langen Sanierungszyklen von bis zu 75 Jahren, die damit der zeitlichen Logik politischer Zielvorgaben hinterherhinken (Michelsen 2015: 920 f.). So gab es in vielen Städten Ostdeutschlands nach der Wende bis etwa ins Jahr 2005 einen Sanierungsschub, in dem die Wärmenachfrage um 20-25 Prozent gesunken ist. Da ein Großteil des Gebäudebestands im Zuge dessen bereits energetisch saniert wurde, sinkt die Nachfrage seitdem nicht mehr so deutlich. Erst wenn hier ein neuer Sanierungszyklus ansteht, ergeben sich Chancen für den Umbau der Wärmeversorgung (Interview A).

Anders als der Heizwärmebedarf steigt der Energiebedarf für Raumklimakälte. In Deutschland wurde in den letzten Jahren ein Zuwachs um 15 Prozent verzeichnet. ${ }^{1}$ Neben der Zunahme von Hitzeperioden, die insbesondere in dicht bebauten Stadtgebieten spürbar werden, sowie dem vermehrten Einsatz transparenter Bauteile und höheren Wärmelasten in Gebäuden durch technische Geräte spielen hier auch wachsende Komfortansprüche eine entscheidende Rolle (Henning/Urbaneck/Morgenstern et al. 2009: 9).

Deutlich wird, dass im Wärmemarkt ein tief greifender Wandel notwendig ist und die vorhandene Infrastruktur unter einem hohen Anpassungsdruck steht. Die Entwicklun-

\footnotetext{
${ }^{1}$ Petersen, S. (2012): Absorptionskälteanlagen für die Gebäudekühlung - Projektergebnisse aus EnEff Wärme. Vortrag auf dem 17. Dresdner Fernwärme-Kolloquium, 19.09.2012.
} 
Abb. 2 Entwicklung der beheizten Flächen und des spezifischen Heizwärmeverbrauchs (Nitsch/Pregger/Naegler et al. 2012: 65)

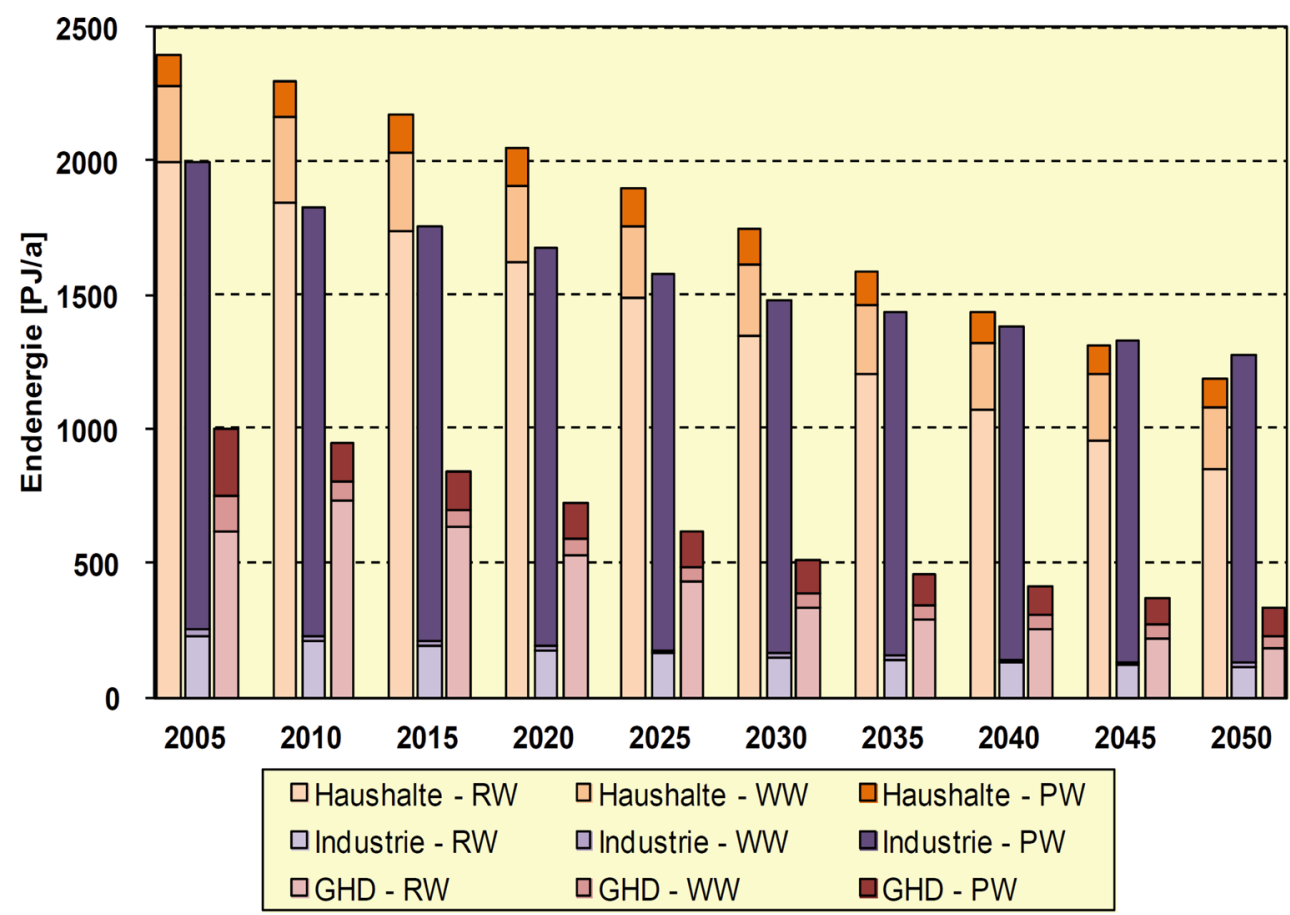

gen des Marktes und die Ziele der Energiewende für diesen weisen aber insbesondere mit dem Ziel einer Reduktion der Energienachfrage und gleichzeitig einer zunehmenden Versorgung über Wärmenetze Zielkonflikte auf. Zugleich steht der Wärmemarkt sowohl technisch als auch ökonomisch in vielfältiger Verbindung zum Strommarkt und auch einem wachsenden Kälteenergiebedarf. Diese Interdependenzen machen sowohl die Ziele vor dem Hintergrund der Energiewende als auch die Strategien, die den unterschiedlichen Zielen, Entwicklungen und Abhängigkeiten Rechnung tragen, komplex.

\section{Zwischen Unvereinbarkeiten und Synergien konkurrierender Strategien}

Die Betrachtung einer möglichen Konkurrenz oder Koexistenz von netzgebundenen Wärmeversorgungsstrategien und der Reduktion der Energienachfrage durch energetische Sanierung lässt sich einbetten in die sozialwissenschaftliche Technikforschung, deren Debatten Hinweise zur Analyse und Interpretation des Verhältnisses zwischen beiden Strategien liefern. Zudem wurde eine mögliche Konkurrenz bereits in der wissenschaftlichen Literatur diskutiert und unterschiedliche Ansätze eines Umgangs damit aufgezeigt.

Hughes, ein Begründer der sozialwissenschaftlichen Technikforschung, hat in seinen Arbeiten großtechnische Systeme charakterisiert und ihre Eigenschaften, ihr Entstehen und ihre Entwicklung untersucht. Zentral in seinen Untersuchungen sind Beharrungstendenzen, die durch die
Eigendynamik großtechnischer Systeme entstehen; diese sind ihrer hohen Kapitalintensität und Komplexität, aber auch der Kopplung ihrer einzelnen Komponenten geschuldet. Dies führt zur „Behäbigkeit“ gegenüber Innovationen und zu Pfadabhängigkeiten in ihrer Weiterentwicklung (Hughes 1987: 76 f.). Aus dieser „Behäbigkeit“ und Innovationsfeindlichkeit heraus kann es zu einem möglichen „battle of the systems" kommen, wenn das System sich nicht aus sich heraus ausreichend weiterentwickeln und an neue Herausforderungen anpassen kann. Stattdessen kommt ein neues System hinzu, tritt in Konkurrenz zu dem etablierten System und fordert dieses heraus. In diesem Wettbewerb setzt sich schließlich eines der beiden Systeme durch und verdrängt das andere (Hughes 1987: 77). Eine solche Konkurrenz ist gerade bei großtechnischen Infrastruktursystemen relevant, da aufgrund ihrer oben genannten technischen und ökonomischen Eigenschaften (Kapitalintensität, Komplexität, Netzgebundenheit und Kopplung der Komponenten) in der Regel nicht mehrere Systeme parallel wirtschaftlich betrieben werden können.

Dennoch lassen sich Hughes' Arbeiten und das Konzept eines „battle of the systems“ nicht direkt auf die hier betrachtete Fragestellung übertragen. So hat er seine Erkenntnisse am Beispiel der Elektrizitätsversorgung erarbeitet. Der Wärmemarkt unterscheidet sich grundsätzlich davon und ist geprägt von einer Vielfalt verschiedener Versorgungslösungen mit unterschiedlichen Techniken und Energieträgern, die im Wettbewerb zueinander stehen, aber dennoch gleichzeitig existieren, ohne dass das eine das andere in jedem Fall verdrängen muss. Dieses wird beispielsweise an den 
Gas- und Fernwärmenetzen deutlich, die für ihre ökonomische Betriebsweise beide möglichst hohe Anschlussraten benötigen, aber dennoch in zahlreichen Stadtquartieren parallel existieren. Der Tatsache, dass verschiedene technische Lösungen auch gleichzeitig existieren können, eine neue Technik eine alte überlagert, ohne dass diese verdrängt wird, oder auch mehrere Systeme gleichwertig bestehen, wurde auch in der sozialwissenschaftlichen Technikforschung Rechnung getragen, indem von verschiedenen „Layern“ (Schichten) ausgegangen wird, die einander überlagern und koexistieren (Henckel/Moss 2015). Dieser Ansatz ist aber ebenfalls nicht direkt auf die hier beschriebene Situation übertragbar, denn betrachtet man zusätzlich das Ziel einer Reduktion des Energiebedarfs durch energetische Sanierung und damit die Nachfrageseite, so handelt es sich nicht in diesem Sinne um ein mit Wärmenetzen konkurrierendes System. Stattdessen sind es zwei verschiedene Strategien im Zuge des durch die Ziele der Energiewende anvisierten Umbaus der Wärmeversorgung, deren Kompatibilität zumindest in der Literatur in Frage gestellt wurde (Späth 2005: 343). So hat beispielsweise Magnusson (2012) am Beispiel des schwedischen Fernwärmesektors gezeigt, wie dieser durch die sinkenden Verbräuche aufgrund der verbesserten Gebäudeenergieeffizienz stagnierte und zunehmend unter ökonomischen Druck geriet (Magnusson 2012: 453). Vor dem Hintergrund der energiepolitischen Zielsetzungen, mit der beide Strategien verfolgt werden, spricht Späth deshalb von ,interfering strategies“ (interferierenden, d. h. sich gegenseitig behindernden oder beeinträchtigenden Strategien) auf dem Weg zur nachhaltigen Energieversorgung (Späth 2005: 339).

Tatsächlich ist dieser Wettbewerb zwischen den unterschiedlichen Versorgungslösungen und Effizienzstrategien auf der Nachfrageseite aber deutlich eingeschränkt. Gebäudeeigentümer investieren größere Summen in die Heizungsanlage und binden sich damit für einige Zeit an eine Technik und einen Energieträger. Bei netzgebundenen Systemen sind auch die Investitionskosten für die Energieversorger sehr hoch und in einigen Gebieten bestehen Anschlusszwänge, sodass die Nutzer an das Versorgungssystem für einen längeren Zeitraum gebunden sind. Hier machen sich die durch die hohe Kapitalintensität verursachten Pfadabhängigkeiten großer und insbesondere netzgebundener Infrastruktursysteme bemerkbar, wie sie unter anderem von Hughes beschrieben wurden. Auch Späth thematisiert in diesem Zusammenhang die Problematik der ,sunk costs“, die zu einem „technological lock-in“ (Späth 2005: 342) führen. Dieser „lock-in“ - verursacht durch die auch von Hughes beschriebenen Beharrungstendenzen großtechnischer Systeme - gilt dabei nicht nur für das Versorgungssystem als solches. Durch dessen Angewiesenheit auf einen notwendigen Mindestverbrauch bestehen auch Abhängigkeiten zu der für die Wärmenachfrage benötigten Dichte und damit zur Siedlungs- und Gebäudestruktur. Die räumlichen Strukturen sind damit nicht nur Einflussfaktor für die Wahl und Gestaltung des Infrastruktursystems, sondern dieses schafft auch andersherum Pfadabhängigkeiten hinsichtlich der Raumstruktur.

Andererseits sind Wärmenetze sehr unterschiedlich. Es gibt kein homogenes System und jedes Netz hat seine eigenen technischen Parameter mit unterschiedlichen Folgen für die benötigte Nachfragedichte und die Nutzungsmöglichkeiten verschiedener Energieträger zur Wärmeerzeugung. Neben zentralen Fernwärmenetzen auf fossiler Basis gibt es zunehmend kleinere Nahwärmenetze, die die Wärme aus dezentralen, oft gasbetriebenen Blockheizkraftwerken oder aus erneuerbaren Wärmequellen wie Energieholz, Grundwasser oder Erdwärme nutzen und verteilen. Die Abgrenzung zwischen Fern- und Nahwärme ist dabei unscharf und wird teilweise an der Länge des Netzes, teilweise aber auch an der Größe der Erzeugungseinheiten oder der Anzahl der Nutzer festgemacht. Pragmatisch wird eine Obergrenze für Nahwärme in der Literatur bei etwa einem Kilometer Transportentfernung festgelegt (Fischedick/Schüwer/ Venjakob et al. 2006: 63).

Gerade wenn in einem Nahwärmenetz erneuerbare Energien wie Umweltwärme oder Niedertemperaturabwärme zum Einsatz kommen, wird die Wärme auf einem deutlich niedrigeren Temperaturniveau verteilt als es bei klassischen Fernwärmenetzen üblich ist. Diese Wärmequellen werden über Wärmepumpen auf das benötigte Temperaturniveau gehoben und sind für ihre ökonomische und ökologische Nutzbarkeit auf einen möglichst geringen Wärmebedarf und, ganz im Gegensatz zu den Fernwärmenetzen auf hohem Temperaturniveau, auch auf möglichst energieeffiziente Gebäude angewiesen (Kaltschmitt/Streicher 2009). Damit eignen sich verschiedene Gebäude- und Nutzungsstrukturen unterschiedlich gut für verschiedene Arten von Wärmenetzen, und andere Siedlungsstrukturen sind aufgrund geringer Dichte ungeeignet für eine netzgebundene Versorgung. Zwischen der Stadtstruktur, ihren Gebäuden, Nutzungseigenschaften und dem Wärmenetz ist damit immer eine jeweils spezifische Abstimmung herzustellen. So wie keine Stadtstruktur genau der anderen gleicht, sind auch alle Netze in ihren Eigenschaften unterschiedlich.

Daher betonen Pehnt/Paar/Otter et al. (2009), dass es keine in jedem Fall gültige Konkurrenz oder Inkompatibilität zwischen Wärmenetzen einerseits und Effizienzsteigerung auf der Verbrauchsseite durch energetische Sanierung andererseits gäbe, sondern dass diese beiden Strategien in manchen Fällen kompatibel und geradezu aufeinander angewiesen sind. Denn auch wenn die Zukunftsfähigkeit zentraler Fernwärmenetze aufgrund der hohen Kapitalintensität und angesichts reduzierter Verbräuche von vielen Autoren in Zweifel gezogen wird, so werden für kleinere Nahwärmenetze aufgrund der geringeren Verluste und Trassenlän- 
gen und der guten Möglichkeit zur Integration erneuerbarer Energien sowohl klimapolitisch als auch ökonomisch gute Chancen gesehen. Teilweise wird sogar die Möglichkeit diskutiert, Gebäude mit sehr geringem Wärmeenergiebedarf über den Rücklauf konventioneller Fernwärmenetze zu versorgen (Pehnt/Paar/Otter et al. 2009: 22 ff.). So könnten auch sehr energieeffiziente Gebäude für eine Versorgung über Wärmenetze geeignet sein, allerdings sind in jedem Einzelfall die Ziele der Gebäudeenergieeffizienz und die Nutzung erneuerbarer Energien bei der Fernwärmeversorgung sinnvoll in Einklang zu bringen.

Statt eine der beiden Strategie zuungunsten der jeweils anderen zu forcieren, müsse daher eher die Balance in jedem Einzelfall gefunden werden (Pehnt/Paar/Otter et al. 2009: 22 ff.). Dass aber dennoch eine relativ hohe Bedarfsdichte notwendig ist, um die netzgebundene Verteilung ökonomisch und ökologisch zu rechtfertigen, bleibt ein wichtiges Thema. In der Balance zwischen Nachfrage und Versorgung können daher je nach der Struktur des Netzes, seinen Eigenschaften und der zukünftigen Verbrauchsentwicklung Probleme zwischen der Strategie der netzgebundenen Versorgung und sinkender Energienachfrage entstehen (Fischedick/Schüwer/Venjakob et al. 2006: 158).

Aus den in der wissenschaftlichen Literatur ausgetauschten Argumenten lassen sich drei Formen ableiten, wie sich eine mögliche Konkurrenz oder Koexistenz zwischen der Nutzung bzw. dem Ausbau von Wärmenetzen und Vorhaben der energetischen Sanierung zeigen kann und wie konkret auf der Umsetzungsebene mit dem Aufeinandertreffen beider Strategien umgegangen werden kann.

- Unvereinbarkeit: Die Zielkonflikte zwischen beiden Strategien werden nicht aufgelöst; bestehende und neu geschaffene Pfadabhängigkeiten werden deutlich.

- Anpassungen: Die Zielkonflikte beider Strategien werden durch Maßnahmen auf der Versorgungs- oder Nachfrageseite nicht aufgehoben, aber entschärft.

- Koexistenz und Synergien: Die Zielkonflikte kommen nicht zum Tragen und eine Koexistenz und sogar Synergien zwischen beiden Strategien sind möglich.

\section{Kommunale Fallbeispiele zum Umgang mit konkurrierenden Strategien}

Wie sich diese verschiedenen Konstellationen des Aufeinandertreffens von energetischen Sanierungsvorhaben und Strategien netzgebundener Wärmeversorgung auf kommunaler Ebene zeigen, soll im Folgenden im Zentrum stehen. Die Beobachtungen aus vier städtischen Fallstudien werden dafür anhand der drei zuvor definierten Formen strukturiert, die dieses Aufeinandertreffen beschreiben können.

Die Beispiele stammen aus Fallbetrachtungen einer deutschen Großstadt und dreier Schweizer Großstädte, die auf unterschiedliche Weise den Umbau ihrer Wärmeversorgung im Hinblick auf deren Klimaverträglichkeit vorantreiben und dabei verschiedene Wege gehen, um mit möglichen Zielkonflikten und Synergien zwischen den beschriebenen Strategien umzugehen. Tab. 1 (oben) gewährt einen Überblick über die vier Beispielstädte und die jeweils wichtigsten Strategien im Zuge des Umbaus ihrer Wärmeversorgung.

\subsection{Unvereinbarkeit der Strategien}

Die Inkompatibilität von netzgebundenen Wärmeversorgungsstrategien und energetischer Sanierung zeigt sich an mehreren Beispielen. Besonders deutlich wird die Unvereinbarkeit der Zielsetzungen, wenn die aktuell hohen und auch für die Zukunft als konstant eingeschätzten Wärmeenergiebedarfe des Bestands als zentrales Argument für den Erhalt und den Ausbau von zentralen Fernwärmenetzen herangezogen werden. Dabei wird dieser Zielkonflikt von den beteiligten Akteuren in der Regel nicht als Problem definiert, da oftmals aufgrund der baulichen Situation ohnehin von nur begrenzten Sanierungsmöglichkeiten ausgegangen wird. In diesen Fällen handelt es sich überwiegend um eine innerstädtische, dichte Bebauung aus der Gründerzeit und teilweise sogar um mittelalterliche, kleinteilige Altstadtstrukturen, die in Teilen denkmalgeschützt sind.

So setzt beispielsweise die Stadt Bern auf einen umfangreichen Ausbau der Fern- und Nahwärme bei gleichzeitigem Rückbau des Gasnetzes (Stadt Bern, Direktion für Sicherheit, Umwelt und Energie 2012: 36). Der Schwerpunkt der Fernwärmeausbauplanungen liegt dabei in den Quartieren, in denen aufgrund der Erhaltung der historischen Altstadt mit UNESCO-Weltkulturerbestatus die Potenziale zur Senkung der Energienachfrage durch energetische Sanierung als gering eingeschätzt werden (Interview B). Hier wird mit einer langfristig konstanten und relativ hohen Nachfrage gerechnet, auf die das Netz ausgelegt wird. In der Gesamtbetrachtung der konkreten baulichen Situation und ihren Anforderungen an die Wärmeversorgung erscheint dies ökonomisch und ökologisch sinnvoll. Zudem handelt es sich um ein innerstädtisches Gebiet mit gemischter Nutzung, das aufgrund seiner Bebauungsdichte und seiner Lage geeignet für eine Versorgung über ein auf hohe Verbräuche angewiesenes Fernwärmenetz ist. Dennoch werden hier auch Pfadabhängigkeiten geschaffen, die einen hohen Wärmebedarf für das Netz festlegen, selbst wenn einmal bessere Möglichkeiten der energetischen Sanierung gefunden werden sollten. Grundsätzlich wird damit ein Zielkonflikt zwischen Energiebedarfsreduktion und Wärmeversorgungsstrategien über Netze nicht aufgehoben. Zudem entstehen neue Abhängigkeiten, da die Wärme für das Netz zu großen Teilen aus der Abfallverbrennung stammt und schon heute Probleme mit zu geringen Abfallmengen bestehen (Inter- 
view F). Wird durch den Bedarf der Wärmenetze die Nachfrage nach Abfall zur Verbrennung aber auf hohem Niveau stabilisiert, entstehen Konflikte mit den Zielen der Abfallwirtschaft und damit zwischen den Infrastruktursektoren.

Mit einer ähnlichen Argumentation wie in Bern planen auch die Akteure der Energieversorgung und der Energiepolitik in Frankfurt am Main den Ausbau des zentralen Fernwärmenetzes. Eingebunden sind diese Ausbauplanungen in eine ,spartenübergreifende Zielnetzplanung“, die vom städtischen Energieversorger Mainova gemeinsam mit der TU München durchgeführt wurde und die alle städtischen Energienetze (Gas, Fernwärme und Elektrizität) gemeinsam betrachtete. Im Rahmen dieser Gesamtbetrachtung wurden Bestandsquartiere definiert, die bisher über Gas versorgt sind und mittelfristig an die Fernwärme angeschlossen werden sollen. Nach einem Leitfaden der Stadt Frankfurt am Main sind langfristig für alle Gebiete mit einer Wärmebedarfsdichte von mehr als $50 \mathrm{GWh} / \mathrm{km}^{2}$ Fernwärmelösungen wirtschaftlich zu realisieren und Gas ist nur in Gebieten mit $\leq 30 \mathrm{GWh} / \mathrm{km}^{2}$ vorzuziehen. Zwischen 30 und $50 \mathrm{GWh} / \mathrm{km}^{2}$ sind Nahwärmenetze mit Blockheizkraftwerk als die geeignete Lösung definiert, teilweise können diese auch bei über $50 \mathrm{GWh} / \mathrm{km}^{2}$ eingesetzt werden (Stadt Frankfurt am Main 2014: 5). Für die Betrachtung des Netzes wird als zusätzlicher Schwellenwert ca. 1500 kWh Wärmeabnahme je Meter Trassenlänge und Jahr genannt (Pfister/Wallraff/ Sieverding 2015).

Bei den Quartieren, die auf dieser Grundlage von Gas auf Fernwärme umgestellt werden sollen, handelt es sich überwiegend um Gründerzeitquartiere, in denen keine komplette Fassadendämmung angestrebt werden soll und daher langfristig mit einem hohen Wärmebedarf gerechnet wird (Interview D). In der detaillierten Gesamtbetrachtung des Stadtraums, der Ausgangslage der verschiedenen Baustrukturen und der langfristigen Planungen für die verschiedenen Energienetze ist dieses eine verständliche Planungsgrundlage, für die es ökonomische Argumente gibt und die auch mit den politischen Zielen der Energiewende begründbar ist, insbesondere wenn langfristig eine Alternative zur Verbrennung von Gas in Einzelfeuerungen gefunden werden muss. Ein möglicher Konflikt zwischen den Strategien der verbesserten Gebäudeenergieeffizienz und netzgebundener Wärmeversorgung wird dennoch nur scheinbar aufgelöst, denn die höheren Bedarfe des Altbaubestands bleiben das wichtigste Argument für zentrale Wärmenetze.

\subsection{Anpassungsstrategien}

Trotz der hinter den politischen Zielsetzungen zurückbleibenden Sanierungsrate wurden in den vergangenen Jahren Erfolge in der energetischen Sanierung des Gebäudebestands erzielt und die Wärmenachfrage in den betrachteten Beispielstädten ist in vielen Quartieren gesunken (In- terviews E und H; vgl. Regierungsrat des Kantons Basel Stadt 2009: 128). Dennoch zeigen sich in den kommunalen Fallstudien nicht nur Konfliktlinien zwischen dem Ziel einer Reduktion des Energiebedarfs und netzgebundener Versorgung, sondern es werden auch Strategien der Anpassung an diese Entwicklung deutlich.

So hat sich in Winterthur in den vergangenen Jahren der Rückgang der Energienachfrage für die Raumwärmeversorgung bereits deutlich abgezeichnet. Dennoch setzt die Stadt für ihre zukünftige Wärmeversorgung auch auf ein bereits bestehendes Fernwärmenetz, das überwiegend aus der Abfallverbrennung gespeist wird. Dieses soll, um die Rückgänge in der Nachfrage zu kompensieren, an den Rändern ausgebaut und vor allem im bestehenden Netz verdichtet werden (Interview E). Dafür soll langfristig das Gasnetz im auch von Fernwärme erschlossenen Gebiet zurückgebaut werden, damit langfristig nicht zwei teure, parallel bestehende Wärmeversorgungssysteme unterhalten werden müssen. So soll die Anschlussrate des Fernwärmenetzes erhöht werden, damit auch bei geringerer Wärmenachfrage der einzelnen Kunden das Netz weiterhin ökonomisch betrieben werden kann. Durch diese Maßnahmen, die sich vor allem auf den Anschluss innerstädtischer, gemischt genutzter Quartiere konzentrieren (beispielsweise ein durch Konversion eines alten Industriegebiets entstandenes Wohnund Geschäftsviertel), konnte der Wärmeabsatz zumindest konstant gehalten werden (Interview E). Handlungsspielräume für solche Nachverdichtungen im innerstädtischen Bereich ergeben sich hier aber vor allem, wenn größere Entwicklungen oder Konversion anstehen.

Neben der Anschlussverdichtung im Netz und Ausbau an den Netzrändern ist die Temperaturabsenkung im Netz eine wesentliche Anpassungsstrategie an sinkende Wärmenachfrage. So lässt sich in Bern beobachten, dass in Neubauquartieren mit geringeren spezifischen Verbräuchen neben dem Anschluss größerer Verbraucher und insbesondere von Mehrfamilienhäusern für die Sicherstellung einer ausreichenden Nachfragedichte ein vergleichsweise niedriges Temperaturniveau von 70 Grad die wesentliche Antwort auf die sinkenden Verbräuche ist (Interview F).

Auch Basel reagiert mit einer Absenkung des Temperaturniveaus im Fernwärmenetz auf die rückläufige Wärmenachfrage. Dies geschieht schrittweise und soll bis $2025 \mathrm{im}$ gesamten Basler Fernwärmenetz realisiert sein. Als Pilotgebiet für diese Maßnahme wurde ein Quartier ausgewählt, das am Rande des Netzes liegt und durch einen eigenen Hauptstrang erschlossen ist. Während damit einerseits der veränderten Nachfrage Rechnung getragen wird, entstehen andererseits neue Zielkonflikte, denn einige Kunden beziehen Dampf aus dem Fernwärmenetz, auch als Energie für industrielle Prozesse (Prozessenergie) oder als Antrieb für Kälteerzeugung über Absorptionstechnik. Diese können mit einem niedrigeren Temperaturniveau nicht mehr 
versorgt werden und müssen ihre Prozess- und Kälteenergie anders erzeugen (Interview G). Das Pilotgebiet zeichnet sich aber insgesamt durch eine vergleichsweise homogene Gebäude- und Nutzungsstruktur aus. Neben der überwiegenden Wohnnutzung in relativ dichter Bebauung gibt es nur wenige industrielle Kunden, sodass insgesamt wenig technische Anpassungen nötig sind. Dennoch müssen auch hier bei einigen Kunden Anpassungen an Wärmetauschern vorgenommen werden. Durch das geringere Temperaturniveau können günstigere Rohre eingesetzt werden und es geht weniger Energie verloren. Etwa 40 Prozent der Leitungskosten sollen so in Basel eingespart werden und die geringeren Wärmeabsätze ausgleichen (Interview H).

Auch in Frankfurt am Main gerät das Fernwärmenetz, trotz der oben beschriebenen Ausbauplanungen, unter Anpassungsdruck. Denn auch hier ist die Nachfrage in einigen Bereichen rückläufig und es gelingt nicht, diese Rückgänge durch neue Anschlüsse auszugleichen, auch aufgrund der Dominanz der Gasversorgung (Interview C). Daher soll das Fernwärmenetz dort, wo es noch auf Dampf basiert (vor allem in Teilbereichen der Innenstadt), auf ein Heißwassernetz umgestellt werden, um die Verluste zu verringern und Kosten einsparen zu können. Ähnlich wie in Basel entstehen aber auch in Frankfurt im Zuge der Absenkung des Temperaturniveaus neue Zielkonflikte. So soll in einigen Bereichen die Temperatur im Netz auch erhöht werden mit dem Ziel, eine Kälteerzeugung über Absorptionskälteanlagen realisieren zu können. Durch diese Projekte soll die Wärme auch im Sommer besser ausgenutzt werden (Interview D). Andererseits steht dies im Widerspruch zur Anpassung an geringere Verbräuche für Heizwärme im Winter.

\subsection{Koexistenz der Strategien und Synergien}

Neben Beispielen, in denen eine klare Konkurrenz zwischen Effizienzstrategien auf der Verbrauchsseite und einer Ausschöpfung der Potenziale von Wärmenetzen deutlich wird bzw. diese durch Anpassungen entschärft, aber nicht aufgehoben werden kann, werden auf kommunaler Ebene auch verschiedene Konstellationen deutlich, in denen beide Strategien zusammen umgesetzt werden, da sich Synergien ergeben und beide sogar aufeinander angewiesen sind.

Hier sticht Frankfurt am Main besonders hervor, da die Stadt schon seit vielen Jahren parallel zum Schwerpunkt beim Ausbau von Wärmenetzen auch die energetische Sanierung des Bestands vorantreibt. Schon seit Beginn der 1990er-Jahre setzt Frankfurt stark auf die Förderung dezentraler Kraft-Wärme-Kopplung und den Ausbau von Fernund Nahwärmenetzen (IFEU 2008: 60). Gleichzeitig gilt die Stadt als Vorreiterin im energieeffizienten Bauen und wurde schon früh als „Passivhaushauptstadt“ (Petermann 2011) bezeichnet. So führte Frankfurt schon 1992 einen Energiepass ein, der auf ähnlichen Bilanzierungsmethoden basierte wie die spätere Energieeinsparverordnung (Stadt Frankfurt am Main, Dezernat Umwelt und Gesundheit und Dezernat Planen, Bauen, Wohnen und Grundbesitz 2007: 12 f.). Seit 2007 müssen zudem alle städtischen Gebäude bei Neubau und Sanierung dem Passivhausstandard entsprechen (Schumacher/Stroh/Schurig et al. 2015: 157). Dabei scheint in der Frankfurter Energiepolitik kein Konflikt zwischen diesen beiden Strategien zu bestehen. So wurden in Frankfurt seit 1990 fast alle Neubauquartiere an die Fernwärme angeschlossen oder erhielten ein eigenes Nahwärmenetz (Stadt Frankfurt am Main, Dezernat Umwelt und Gesundheit und Dezernat Planen, Bauen, Wohnen und Grundbesitz 2007: 17). Darunter waren auch Passivhäuser, deren wirtschaftliche Erschließung mit Wärmenetzen auf niedrigem Temperaturniveau realisiert werden konnte (Interview D). Die Schwelle für die Wärmebedarfsdichte von $30 \mathrm{GWh} / \mathrm{km}^{2}$ (s. oben) wird trotz der hohen Energieeffizienz der Gebäude aufgrund einer vergleichsweise dichten Bebauung erreicht (Stadt Frankfurt am Main 2014: 13 ff.).

Gleichzeitig sollen in geeigneten Stadtquartieren neue Nahwärmeverbünde eingerichtet werden, die erneuerbare Energiequellen nutzen, wie beispielsweise Holzfeuerungen, Grundwasser oder Erdwärme. Das wesentliche Argument der Stadt zur Einrichtung neuer Wärmenetze ist eine höhere Flexibilität in der Versorgung. So sollen die Netze dazu beitragen, Wärme und gegebenenfalls auch Kälte besser speichern zu können und damit innerhalb des versorgten Quartiers unterschiedliche Bedarfe und Schwankungen in der Nachfrage besser ausgleichen zu können. Zudem sollen die Wärmenetze größtmögliche Flexibilität gewähren, indem sie durch unterschiedliche Energieträger gespeist werden können. Denn gerade aufgrund der langen Lebensdauer der Netze kann heute noch nicht mit Sicherheit gesagt werden, welche Energieträger langfristig eingesetzt werden (Interview C; Paar/Herbert/Pehnt et al. 2013: 64).

Diese Überlegungen spiegeln sich auch in Winterthur wider, wo vermehrt Holz für die Wärmeerzeugung zum Einsatz kommt. Dieses könnte aber zu einem späteren Zeitpunkt gegebenenfalls nur noch als Energieträger für hohe Energiebedarfe (wie beispielsweise Prozesswärme) zum Einsatz kommen, da es auch hohe Temperaturen erzeugen kann (Interview I). Vor dem Hintergrund dieser Überlegungen werden die Nahwärmenetze für viele Wohnquartiere heute auf ein niedriges Temperaturniveau ausgelegt, um neben der Holzverbrennung auch für die Wärmeeinspeisung aus Niedertemperaturwärmequellen wie Grundwasser, Abwasser oder Erdwärme geeignet zu sein, die über Großwärmepumpen auf das benötigte Temperaturniveau gebracht werden. Die Umsetzung solcher Wärmeversorgungskonzepte setzt eine hohe Gebäudeenergieeffizienz voraus, da der Energieaufwand für die Wärmepumpen ansonsten zu groß wäre. An dieser Stelle besteht somit kein Konflikt mit einer Effizienzstrategie auf der Nachfragesei- 
Abb. 3 Wärmenetze und energetische Sanierung zwischen Konkurrenz und Koexistenz



te - im Gegenteil. Zudem sollen einheitliche technische Parameter es ermöglichen, die einzelnen Verbünde zu einem späteren Zeitpunkt gegebenenfalls zu vernetzen und so auch Energieflüsse stadtweit besser ausbalancieren zu können (Stadt Winterthur, Umwelt und Gesundheitsschutz 2011: 46).

Auch in Bern sind Überlegungen zur Flexibilität in der Wärmeerzeugung das zentrale Argument für die Strategie netzgebundener Wärmeversorgung. Der Hintergrund sind Potenzialabschätzungen, die für die zukünftige Wärmeversorgung gute Chancen in der Nutzung von Abwärme, Sonnenenergie, Erdwärme über Wärmepumpen, energieholzbasierten Heizkraftwerken und im noch unsicheren Potenzial aus Tiefengeothermie sehen (Interview B). Durch die Etablierung von Nahwärmeverbünden soll die Möglichkeit geschaffen werden, die Einspeisung unterschiedlicher erneuerbarer Energieträger offenzuhalten. Allerdings sind viele dieser Energieträger nur auf einem niedrigeren Temperaturniveau verfügbar; wenn sie langfristig die Wärmeversorgung sicherstellen sollen, sind sie auf entsprechend geringe Nachfragen und energieeffiziente Gebäude angewiesen. Das Ziel dieser Planungen ist, dass die fossilen Energieträger bis 2035 nur noch 20 Prozent zum Wärmemix der Stadt beitragen (Stadt Bern, Direktion für Sicherheit, Umwelt und Energie 2012: 22, 31, 37).

Deutlich wird aus den Betrachtungen der kommunalen Beispiele, dass eine Konkurrenz zwischen energetischer Sanierung und dem Auf- bzw. Ausbau von Wärmenetzen bestehen kann; diese Konkurrenz wird aber in vielen Fällen durch Anpassungsstrategien abgemildert oder es werden sogar Synergien zwischen beiden gefunden, insbesondere wenn erneuerbare Energien in Nahwärmenetzen zum Einsatz kommen. Die gewählten Strategien für das Aufeinandertreffen von Versorgungslösungen und energetischer Sanierung sind dabei eng mit den konkreten raum- und siedlungsstrukturellen Charakteristika der versorgten Gebiete verknüpft. Gerade bei neuen Entwicklungen oder großen umfangreichen Sanierungen wie beispielsweise im Zuge einer Konversion entstehen Handlungsspielräume, um an den (gegebenenfalls reduzierten) Bedarf angepasste Wärmenetze, teilweise auch auf Basis erneuerbarer Energien, zu etablieren.
Abb. 3 fasst die wesentlichen Ergebnisse aus den Fallstudien zum Aufeinandertreffen von Strategien zum Ausbau von Wärmenetzen und energetischer Sanierung zwischen Konkurrenz und Koexistenz zusammen.

\section{Zusammenfassung und offene Fragen}

Die klimapolitischen Ziele für die Optimierung der Wärmeversorgung sind hoch gesteckt und ihre Erreichung ist zentral, damit die Treibhausgasemissionen entscheidend gesenkt werden können. Städte und ihr Gebäudebestand spielen bei der Transformation der Wärmeversorgung eine zentrale Rolle und sind sowohl für die Zielsetzungen und Maßnahmen zur energetischen Sanierung als auch für die Integration erneuerbarer Energien in die Wärmeversorgung wichtige Adressaten. Viele Städte setzen für die zukünftige Wärmeversorgung unter anderem auf Wärmenetze, um langfristig Flexibilität hinsichtlich des Einsatzes erneuerbarer Energieträger zu erreichen.

Zwischen den betrachteten Fallstudien zeigen sich dabei viele Parallelen. So werden in allen vier untersuchten Städten sowohl die Reduktion des Energiebedarfs über energetische Sanierung als auch ein Ausbau und eine Anpassung der Wärmenetze parallel verfolgt. Dabei wird der Zielkonflikt zwischen diesen beiden Strategien oftmals nicht als Widerspruch definiert. Eine Konkurrenz kann nur teilweise im Zuge des Anpassungsdrucks auf die zentralen Fernwärmenetze festgestellt werden. Deutlich wird aus den Fallstudien, dass gerade große Fernwärmenetze mit hohem Temperaturniveau durch die sinkende Nachfrage unter ökonomischen Druck geraten (Bern, Basel, Winterthur). Hier sind Anschlussverdichtungen und Ausbau an den Rändern die wesentlichen Strategien, um die Rückgänge zu kompensieren. Dabei zielt der Ausbau insbesondere auf innerstädtische Gründerzeitquartiere oder verdichtete Wohnquartiere aus den 1960er- und 1970er-Jahren mit hohem Wärmebedarf und teilweise eingeschränkten Sanierungsmöglichkeiten. Dies zeigt, dass oftmals keine Kompatibilität beider Strategien gesehen oder gesucht wird, sondern gerade der hohe Energiebedarf zu einem wesentlichen Argument für die Versorgung über zentrale Netze gemacht wird. Mit Rücksicht auf die Stadt- und Gebäudestrukturen, ihre 
Eignung für netzgebundene Wärmeversorgung und die oftmals ohnehin anfallende Wärme aus der Stromerzeugung oder Müllverbrennung erscheint dieses Vorgehen auch ökonomisch sowie ökologisch sinnvoll. Dennoch werden hier neue Pfadabhängigkeiten geschaffen und Verbräuche ,festgeschrieben“, auf die das Netz auch in Zukunft ausgelegt wird.

Im Gegensatz zu der in der Literatur beschriebenen Inkompatibilität von energetischer Sanierung und der Nutzung bzw. dem Ausbau von Wärmenetzen zeigen sich in der Realität zahlreiche Zwischenwege. So wurde an mehreren Stellen in den Fallstudien deutlich, dass das parallele Verfolgen beider Strategien nicht nur möglich, sondern sogar eine Voraussetzung ist, um in Nahwärmenetzen Wärmequellen aus erneuerbaren Energien auf niedrigem Temperaturniveau (wie beispielsweise Erdwärme oder Wärme aus Grundwasser) über Wärmepumpen nutzbar zu machen. Für die erfolgreiche „Wärmewende“ verfolgen die Städte daher mehrere Lösungswege, die eng mit den unterschiedlichen Raum- und Siedlungsstrukturen verknüpft sind. Während die innerstädtischen, baulich dichten Strukturen mit älterem Gebäudebestand trotz (begrenzter) energetischer Sanierung weiter für die Versorgung über zentrale Fernwärme vorgesehen werden und höchstens über die Absenkung des Temperaturniveaus Anpassungen vorgenommen werden (Frankfurt am Main, Basel), sind gerade in Quartieren aus den vergangenen fünfzehn Jahren oder Neubauquartieren mit hohem Energieeffizienzstandard die Möglichkeiten für Nahwärmenetze unter Nutzung erneuerbarer Energieträger groß (Bern, Winterthur). Hier zeigt sich in den Fallstudien, dass kleinere und hinsichtlich der Energieträger flexible Netze auf niedrigem Temperaturniveau die Antwort auf sinkende Wärmenachfragen sind. Im Bestand aber können sich auch Probleme ergeben, wenn die Nutzer einen zu hohen Energiebedarf haben, um auf niedrigem Temperaturniveau versorgt werden zu können. Angesichts der niedrigen Sanierungsquote muss auch auf der Nachfrageseite aufgeholt werden, um den Anforderungen an die Versorgung mit erneuerbaren Energien entsprechen zu können.

$\mathrm{Zu}$ Beginn des Beitrags wurde die Frage gestellt, ob und inwieweit sich eine Konkurrenz zwischen netzgebundenen Wärmeversorgungsstrategien und der Reduktion der Energienachfrage durch energetische Sanierung zeigt bzw. inwieweit auch eine Koexistenz beider Strategien möglich ist. Aus den Parallelen der Fallstudien lassen sich zentrale Ergebnisse formulieren:

- Eine Konkurrenz zwischen der Nutzung von Wärmenetzen und Vorhaben der energetischen Sanierung kann bestehen, wenn aufgrund der Abhängigkeit der Fernwärme von konstanten Verbräuchen zu schnell von nur begrenzt sanierungsfähigen Altbaubeständen ausgegangen wird und der Zielkonflikt beider Strategien damit nur scheinbar aufgelöst wird.

- Anpassungen der Wärmenetze an sinkende Wärmenachfragen, insbesondere durch Temperaturabsenkung, sind im Zuge der Transformation der Wärmeversorgung eine zwingende Voraussetzung.

- Durch das Erreichen von Synergien werden die Zielkonflikte zwischen energetischer Sanierung und dem Betrieb von Wärmenetzen vielfach aufgelöst. Das parallele Verfolgen beider Strategien ist für die Städte zentral, um die energiepolitischen Ziele für die Wärmewende zu erreichen. In den städtischen Fallstudien wird deutlich, dass Wärmenetze als zentrale Möglichkeit angesehen werden, erneuerbare Energien in die Wärmeversorgung zu integrieren; eine Reduzierung des Energiebedarfs ist hierfür notwendig.

Insgesamt zeigen die Betrachtungen aus den Fallstudien, dass die Wärmeversorgung vielfältig und komplex ins Energiesystem als Ganzes eingebunden ist. Abhängigkeiten und Anpassungsbedarf bestehen nicht nur hinsichtlich des Wärmebedarfs, sondern auch in Bezug auf die Stromproduktion und die Abfallverbrennung als aktuell wichtigen Wärmelieferanten, die wiederum eigenen klimapolitischen, aber auch ökonomischen Zielsetzungen verpflichtet sind. Dabei entstehen neue Zielkonflikte und auch Inkompatibilitäten verschiedener Strategien. Hier bleiben offene Fragen, die die teilweise konträren Zielsetzungen und Innovationshemmnisse angesichts von Pfadabhängigkeiten zwischen den Systemen der Energieversorgung (Gas, Wärme, Strom), aber auch zwischen den Infrastruktursektoren (vor allem mit der Abfallwirtschaft) adressieren.

Auf dem insgesamt schrumpfenden Wärmemarkt konkurrieren verschiedene Systeme und Energieträger. Die übergeordneten klimapolitischen Ziele setzen einen hohen Veränderungs- und Anpassungsdruck, dessen Umsetzung auf kommunaler Ebene noch viele Fragen aufwirft. So legen die Wechselwirkungen der Wärmeversorgung mit der Raum- und Siedlungsstruktur und insbesondere die Balance zwischen Effizienzsteigerungen auf der Nachfrageseite und Anpassungen auf der Versorgungsseite eine tiefer gehende Behandlung des Themas an der Schnittstelle von Energie(fach)planung und Stadtplanung nahe. Auch wenn noch viele Hindernisse überwunden werden müssen und das Tempo der „Wärmewende“ dem in anderen Bereichen des Energieversorgungssystems deutlich hinterherhinkt, können die vielfältigen neuen Lösungen der Wärmeversorgung auf der Basis erneuerbarer Energien und vor dem Hintergrund des Anpassungsdrucks durch die sinkende Nachfrage als innovative Antworten verstanden werden. 
Danksagung Mein herzlicher Dank gilt Prof. Dr. Sabine Hofmeister und Dr.-Ing. Jörg Fromme für ihr konstruktives Feedback bei der Erarbeitung dieses Beitrags.

\section{Verzeichnis der Interviews}

Interview A Leiter Unternehmensentwicklung der Stadtwerke Rostock, 24.10.2013, Rostock

Interview B Leiterin der Sektion Umwelt und Energie der Stadt Bern, 07.05.2013, Bern

Interview C Vertreter des Energiereferats Frankfurt am Main, 29.08.2013, Frankfurt am Main

Interview D Vertreter der Abt. Grundsatzplanung Wärme der NRM Netzdienste Rhein-Main GmbH, 07.10.2013, Frankfurt am Main

Interview E Vertreter der Abt. Wärme und Entsorgung der Stadtwerke Winterthur, 22.04.2013, Winterthur

Interview F Leiter der Abt. Energiedienstleistung Energie Wasser Bern, 21.05.2013, Bern

Interview G Leiter der Abt. Energie des Amts für Umwelt und Energie Basel, 24.04.2013, Basel

Interview H Vertreter der Abt. Fernwärme der Industriellen Werke Basel, 15.05.2013, Basel

Interview I Leiter Energiecontracting der Stadtwerke Winterthur, 26.06.2013, Basel

\section{Literatur}

AG Energiebilanzen (Hrsg.) (2013): Anwendungsbilanzen für die Endenergiesektoren in Deutschland in den Jahren 2011 und 2013 mit Zeitreihen von 2008-2012. http://www.ag-energiebilanzen. de/10-0-Auswertungstabellen.html (15.01.2016).

Berger, T.; Genske, D. D.; Hüsler, L.; Jödecke, T.; Menn, A.; Ruff, A. (2011): Endbericht Energetische Optimierung des Kantons BaselStadt. Nordhausen, Liechtenstein.

Bundesministerium für Wirtschaft und Technologie (Hrsg.) (2010): Energie in Deutschland - Trends und Hintergründe zur Energieversorgung. Aktualisierte Ausgabe. http://www.bmwi. de/BMWi/Redaktion/Bilder/Service/Publikation/energie-indeutschland, property $=$ pdf, bereich $=$ bmwi2012, sprache $=$ de, rwb=true.pdf (15.01.2016).

Fischedick, M.; Schüwer, D.; Venjakob, J.; Merten, F.; Mitze, D.; Krewitt, W.; Nast, M.; Schillings, C.; Bohnenschäfer, W.; Lindner, K. (2006): Anforderungen an Nah- und Fernwärmenetze sowie Strategien für Marktakteure in Hinblick auf die Erreichung der Klimaschutzziele der Bundesregierung bis zum Jahr 2020. http:// www.dlr.de/Portaldata/41/Resources/dokumente/institut/system/ publications/UBA_Nahwaerme_EB_111206.pdf (09.05.2016).

Graichen, V.; Bürger, V.; Gores, S.; Penninger, G.; Zimmer, W.; Eichhammer, W.; Fleiter, T.; Schlomann, B.; Strigel, A.; Ziesing, H.-J. (2012): Energieeffizienzdaten für den Klimaschutz. https://www. umweltbundesamt.de/sites/default/files/medien/publikation/long/ 4343.pdf (15.01.2016).

Henckel, D.; Moss, T. (2015): Towards a brighter future? Conclusions for lighting research and policy. In: Meier, J.; Hasenöhrl, U.; Krause, K.; Pottharst, M. (Hrsg): Urban Lighting, light pollution and society. New York, 299-304.

Henning, H.-M.; Urbaneck, T.; Morgenstern, A.; Nunez, T.; Wiemken, E.; Thümmler, E.; Uhlig, U. (2009): Kühlen und Klimatisieren mit Wärme. Berlin

Hughes, T. P. (1987): The Evolution of Large Technical Systems. In: Bijker, W.; Hughes, T. P.; Pinch, T. (Hrsg.): The Social Construction of Technological Systems: New Directions in the Sociology and History of Technology. Cambridge, 51-82.
IFEU - Institut für Energie- und Umweltforschung Heidelberg GmbH (Hrsg.) (2008): Energie- und Klimaschutzkonzept für die Stadt Frankfurt am Main. https://www.frankfurt.de/sixcms/media.php/ 738/IFEU_KSK_Frankfurt_Endbericht_Aug09_1.pdf (15.01.2016).

Kaltschmitt, M.; Streicher, W. (2009): Regenerative Energien in Österreich. Wiesbaden.

Löschel, A.; Erdmann, G.; Staiß, F.; Ziesing, H.-J. (2014): Stellungnahme zum ersten Fortschrittsbericht der Bundesregierung zum Berichtsjahr 2013. https://www.bmwi.de/BMWi/Redaktion/PDF/ $\mathrm{M}-\mathrm{O} /$ monitoringbericht-energie-der-zukunft-stellungnahme2013, property $=$ pdf, bereich $=$ bmwi2012, sprache $=$ de, $r w b=$ true . pdf (15.01.2016).

Magnusson, D. (2012): Swedish district heating - A system in stagnation: Current and future trends in the district heating sector. In: Energy Policy 48, 449-459.

Michelsen, C. (2015): Wärmemonitor Deutschland 2014: Rückläufiger Energiebedarf und lange Sanierungszyklen, In: DIW Wochenbericht 41, 920-931.

Nitsch, J.; Pregger T.; Naegler, T.; Heide, D.; Luca de Tena, D.; Trieb, F.; Scholz, Y.; Nienhaus, K.; Gerhardt, N.; Sterner, M.; Trost, T.; von Oehsen, A.; Schwinn, R.; Pape, C.; Hahn, H.; Wickert, M.; Wenzel, B. (2012): Langfristszenarien und Strategien für den Ausbau der erneuerbaren Energien in Deutschland bei Berücksichtigung der Entwicklung in Europa und global. Schlussbericht. http://www.dlr.de/tt/Portaldata/41/Resources/dokumente/institut/ system/publications/leitstudie2011_bf.pdf (15.01.2016).

Paar, A.; Herbert, F.; Pehnt, M.; Ochse, S.; Richter, S.; Maier, S.; Kley, M.; Huther, H.; Kühne, J.; Weidlich, I. (2013): Transformationsstrategien von fossiler zentraler Fernwärmeversorgung zu Netzen mit höheren Anteilen erneuerbarer Energien. Endbericht. http://www.eneff-stadt.info/fileadmin/media/Publikationen/ Dokumente/Endbericht_Transformationsstrategien_FW_IFEU_ GEF_AGFW.pdf (15.01.2016).

Pehnt, M.; Paar, A.; Otter, P.; Merten, F.; Hanke, T.; Irrek, T.; Schüwer, D.; Supersberger, N.; Zeiss, C. (2009): Energiebalance - Optimale Systemlösungen für erneuerbare Energien und Energieeffizienz. http://www.energieeffizient-sanieren.org/data/EnergiebalanceEndbericht.pdf (15.01.2016).

Petermann, A. (2011): Passivhaus-Hauptstadt Frankfurt am Main, Website Deutschland Radio Kultur. http://www.dradio.de/dkultur/ sendungen/erneuerbareenergien/1438130/ (17.01.2014).

Pfister, T.; Wallraf, C.; Sieverding, U. (2015): Nahwärmegenossenschaften - Chancen und Risiken aus Verbrauchersicht. http:// www.verbraucherzentrale.nrw/mediabig/235608A.pdf (14.01.2016).

Regierungsrat des Kantons Basel Stadt (2009): Kantonaler Richtplan. http://www.richtplan.bs.ch/ (15.01.2016).

Schumacher, P.; Stroh, K.; Schurig, M.; Ellerbrok, C.; Ramonat, A.; Link, S. (2015): Masterplan 100\% Klimaschutz - Frankfurt am Main - Generalkonzept, http://www.masterplan100.de/ fileadmin/user_upload/content/pdf/generalkonzept/Masterplan Klimaschutz_Generalkonzept_Langfassung.pdf (25.01.2016)

Späth, P. (2005): District heating and passive houses - Interfering strategies towards sustainable energy systems. Proceedings of the ECEEE 2005 summer study. Mandelieu La Napoule, France. http://www.eceee.org/library/conference_proceedings/eceee Summer_Studies/2005c/Panel_2/2057spath/paper (21.01.2016).

Stadt Bern, Direktion für Sicherheit, Umwelt und Energie (Hrsg.) (2012): Richtplan Energie der Stadt Bern. http://www.bern. $\mathrm{ch} /$ themen/umwelt-natur-und-energie/energie/richtplan-energie (09.05.2016). Bern.

Stadt Frankfurt am Main, Dezernat Umwelt und Gesundheit in Zusammenarbeit mit dem Dezernat Planen, Bauen, Wohnen und Grundbesitz (Hrsg.) (2007): Klimaschutz in Frankfurt am Main. https:// www.frankfurt.de/sixcms/media.php/738/Klimaschutzbericht.pdf (09.05.2016). Frankfurt am Main. 
Stadt Frankfurt am Main (Hrsg.) (2014): Aufbau von Wärmenetzen Praxisleitfaden. http://www.energiewende-frankfurtrheinmain. de/fileadmin/user_upload/content/pdf/Anhang_Praxisleitfaeden/ Leitfaden_Aufbau_von_Waermenetzen_Online_Anhang.pdf (14.01.2016).

Stadt Winterthur, Umwelt- und Gesundheitsschutz (Hrsg.) (2011): Grundlagen Energiekonzept 2050. http://ugs.winterthur.ch/fileadmi user_upload/UGS/Dateien/Umwelt/Grundlagen_Energiekonzept_ 2050_Final_20110310.pdf (10.05.2016). Winterthur.
Wolff, D.; Jagnow, K. (2011): Untersuchung von Nah- und Fernwärmenetzen - Überlegungen zu Einsatzgrenzen und zur Gestaltung einer zukünftigen Fern- und Nahwärmeversorgung. https:// www.zukunftsheizen.de/fileadmin/user_upload/3_Technik/3.6_ Projekte_und_Studien/3.6.3_Nah-_und_Fernwaermenetze/Studie_ Untersuchung_Nah-_und_Fernwaermenetze_IWO.pdf (15.01.2016). York, R. (2007): Demographic trends in energy consumption in European Union Nations, 1960-2025. In: Social Science Research 36, $3,855-872$. 